\title{
PREVALENCE OF ONYCHOMYCOSIS IN SOUTH EAST PART OF RAJASTHAN
}

Anita E Chand, Harshad Singh Naruka

1. Professor \& Head, Department of Microbiology, Government Medical College. Kota, Rajasthan

2. Senior Demonstrator, Department of Microbiology, Government Medical College. Kota, Rajasthan

\section{CORRESPONDING AUTHOR}

Mr. Harshad Singh Naruka

H. No. 262, Ward No. 45,

Chhawni ,Kota ,Rajasthan

E-mail: narukaharshadsingh@gmail.com

Ph: 00919829741003

ABSTRACT: Onychomycosis is a fungal infection of nail commonly caused by dermatophytes, yeast and mould. The prevalence of onychomycosis seems to vary across the world because of various socioeconomic and cultural factors. However, all the nail diseases are not fungal in origin Hence laboratory investigations are needed to differentiate accurately between fungal infections and other conditions. MATERIAL AND METHODS: All clinically suspected cases of onychomycosis send to microbiological evaluation at Department of Microbiology Govt. Medical College \& Hospital Kota over a period of one year (January to December 2012) were included in present study. RESULTS: A total of 109 samples (82 male and 27 females) were tested in period of one year (January to December 2012). Among them 55 samples (35 male and 20 females) showed fungal growth, the major pathogen found in present study were Tricophyton spp. 28 (50.9) followed by Candida $10(18.18 \%)$ then Aspergillus spp. 9 (16.36\%), Scopulariopsis 5 (9.09\%), Fusarium 2 (3.63\%) and Alternaria spp 1 (1.81\%). DISCUSSION: - Onychomycosis is a chronic mycotic infection of finger nails and toe nails that affect the quality of life in a significant proportion. There has been a recent increase in the incidence as well as the spectrum of causative pathogens associated with onychomycosis. In our study we found more incidences among men $35(63.63 \%)$ than women (36.36\%). the most common etiology of onychomycosis is dermatophytes (Tricophyton) $50.9 \%$ but there is a considerable increase in Non dermatophytes moulds 17 (30.9\%). For proper management of onychomycosis, diagnosis and accurate treatment play a key role in better outcome.

KEY WORDS: Onychomycosis, Dermatophytes, Non- Dermatophyte moulds

INTRODUCTION: Onychomycosis is a fungal infection of nail commonly caused by dermatophytes, yeast and mould \& sometimes it shows a mixed infection $(1,2)$. The prevalence of onychomycosis is increasing, and the number of organisms recognized as possible fungal pathogens is also increasing. Onychomycosis continues to spread and persist $(2,3)$. The prevalence rate of onychomycosis is determined by age, predisposing factors, social class, occupation, climate, \& living environment. There is a long list of fungi which have a tendency to damage the nail, like dermatophytes (50\%), yeasts $(27 \%)$ and molds $(23 \%)(2,3)$. Dermatophytes, particularly Trichophyton rubrum and Trichophyton mentagrophytes, are the most common cutaneous fungal pathogens $(1,2,3,4,5)$, Non-dermatophyte moulds are fungi with known habitats in soil, decaying plant debris, or plant disease ${ }^{(3,4)}$. They have been traditionally regarded as uncommon or secondary pathogens of already diseased nails $(1,2,3$,$) .The Dermatophytes spp. are$ mostly distributed in the temperate western countries. The Candida spp. (yeasts) is mostly 
distributed in the tropical and the subtropical countries and in the persons whose hands are often submerged in water $(2,3)$. Yeasts include Candida albicans, Candida parapsilosis, Candida tropicalis, etc, while the moulds include a wide range of fungi like Scytalidium spp., Aspergillus spp., Geotrichum candidum and Fusarium spp. $(2,3,4)$. Overall the incidence is much higher in adults than in children (3). The prevalence of onychomycosis seems to vary across the world because of various socioeconomic and cultural factors (3,6). Onychomycosis of finger nail may lead to pain, discomfort, \& impaired/lost tactile functions and the condition has cosmetic, psychological \& physical determinantal effects $(3,6,7)$. However, all the nail diseases are not fungal in origin they are also caused by other clinical conditions like trauma, wet work (with the hand submerged in soap \& water), HIV-AIDS, immunodeficiency which is due to organ or bone marrow transplantation, old age, psoriasis, atopic dermatitis, diabetes with a predominance of Candida spp , renal transplant recipients etc. $(2,3,6)$. Hence laboratory investigations are needed to differentiate accurately between fungal infections and the above mentioned skin diseases for proper management of onychomycosis. Fungal cultures are of paramount importance because the clinical outcome of antifungal agents varies as to whether the etiological agent is a dermatophyte, yeast or mould $(1,2,3,6,7)$.

MATERIAL AND METHODS: All clinically suspected cases of onychomycosis sent to the Department of Microbiology Govt. Medical College \& Hospital Kota for microbiological evaluation by Dermatology Department over a period of one year (January to December 2012) were included in present study. Nail clippings or subungal scrapings from all these subjects were collected with a surgical blade after cleaning the affected area with $70 \%$ ethanol. Microscopic examination of the samples was performed following clarification with $10 \%$ potassium hydroxide (KOH). All samples were inoculated on Sabouraud's dextrose agar (SDA, Hi Media Laboratories) and SDA with 5\% chloramphenicol and cycloheximide. Cultures were incubated at $25^{\circ} \mathrm{C}$ and $37^{\circ} \mathrm{C}$ and examined daily for first week and twice a week for 6 weeks. Different fungi were identified based on microscopic and culture characteristics which were confirmed by slide culture in case of dermatophytes and other moulds, and by germ tube, sugar assimilation test in case of Candida spp. Only those sample which show microscopic evidence of fungi and also grown on culture, considered for further evaluation. The following criteria were taken into consideration for confirming Non-dermatophyte mould as pathogen, on direct microscopic examination large and irregular septate hyphae in $\mathrm{KOH}$ mount, the growth of the same agent in pure culture in at least three tubes of SDA and no growth of dermatophytes as well as repetition of these criteria after an interval of 2 weeks $(3,4,5)$.

RESULTS: A total of 109 samples (82 male and 27 females) were tested in period of one year (January to December 2012). Among them 55 samples 35 male (63.63\%) and 20 females $(36.36 \%)$ showed fungal growth the major pathogen found in present study were Tricophyton spp. 28 (50.9\%) followed by Candida spp. 10 (18.18\%) then Aspergillus spp. 9 (16.36\%), Scopulariopsis 5 (9.09\%), Fusarium 2 (3.63\%) and Alternaria spp 1 (1.81\%). Distribution of various fungi among male and female is as per Table-1

In present study we found that male has more incidence of infection than females. Among total culture positive cases male has $63.63 \%$ infection rate and female has $36.36 \%$. Incidence of Non- dermatophyte moulds are more than Candida and this show that involvement of Non-dermatophyte moulds in onychomycosis is increasing. Among Tricophyton species Tricophyton rubrum showed highest incidence 20 followed by Tricophyton mantegrophytes 7 
and Tricophyton tonsurans 1. Among Candida spp. Candida albicans showed highest incidence 7 followed by Candida tropicalis 3. Among Non-dermatophyte moulds Aspergillus spp showed highest incidence and most common spp. were Aspergillus flavus 7 followed by Aspergillus niger 2, Scopulariopsis spp 5, Fusarium spp. 2, and Alternaria spp. 1. The distribution of various species among male and females are shown in Table -2

DISCUSSION: Onychomycosis is a chronic mycotic infection of finger nails and toe nails that affect the quality of life in a significant proportion (1). The difficulty in diagnosing the condition clinically, inappropriate collection of material for analysis as well as ineffective treatment makes it hard to assess the true profile of such infections $(3,6,7,8)$. There has been a recent increase in the incidence as well as the spectrum of causative pathogens associated with onychomycosis $(1,3,8,9)$ which may be due to increase in awareness among peoples towards health, cosmetic consciousness or due to increase in various risk factors like, chronic diseases leading immunosuppression, other fungal infections of the skin, sport and increased age ${ }^{(10,11,12)}$. It is to note that the treatment of onychomycosis cannot be done only on clinical grounds as we have seen that there are a large number of cases 54 (49.54\%) without any fungal etiology. In our study the onychomycosis was predominant in men 35 (63.63\%) which may be due to more exposure to risk factors, This finding is concordant to various other studies $(1,3,7,8)$ however study from Gujarat (Prakash Gelotar et al., 2012) show high incidence among females (2). It is clear that most common causative agents of onychomycosis are Dermatophytes (Tricophyton) $50.9 \%$. In our study we found much higher incidence of Non-dermatophyte moulds 17 (30.9\%) then other studies (Gupta et al., 2003, Ahuja Sanjiv et al., 2011, Prakash Gelotar et al., 2012 etc) which may be due to variation in geographic differences in mould distribution or diagnostic methods $(3,9)$. In present study age is also found as a determinantal factor in acquiring fungal nail infections, the majority of patient were of age group of 50-60 years $16(29.09 \%)$ followed by age group 60-70 years 14 (25.45\%), then age group 70-80 years 9 (16.36\%), then age group 40-50 years $7(12.72 \%)$ Table-3. Yeasts are also an important cause of onychomycosis and are more frequent in fingernails than toe nails and were second most common cause of onychomycosis in our study. Although not usually life threatening, onychomycosis can be a source of significant pain and discomfort. It can also pose significant risk for patients, their family and others in contact with them $(1,6,10)$. For proper management of onychomycosis, diagnosis and accurate treatment play a key role in better outcome. However several newer diagnostic methods have been introduced like PCR based methods and non invasive methods like optical coherence tomography, confocal laser scan microscopy, matrix assisted laser desorption time of flight mass spectrometry (MALDI-TOF MS) and phase contrast hard x-ray microscopy (8). This may help in early diagnosis and better management of infection? But as the spectrum of disease is based on various etiologies, so the culture will remain gold standard in identifying the species causing the infection, and the fact that such complicated and sophisticated methods will remain limited to some higher centers only. Various treatment strategies including topical, systemic and surgery which is used to treat the condition depends on severity of disease. Despite good therapeutic response the long term outcome is unsatisfactory due to therapeutic failure, relapse and reinfection $(1,3,7,8,11)$. To combat poor response newer strategies such as combination, sequential \& supplementary therapy have been suggested (11). The following are the strategies to improve cure rate in onychomycosis (11) 
- Ensure correct and complete diagnosis

- Choose most appropriate antifungal

- Ensure bioavailability and compliance

- Monitor for any possible drug interactions

- Consider supplemental therapy-in poor prognostic factors

- Consider surgery in addition to antifungal therapy if required

- Education regarding risk factors and nail care

- Ensure treatment of affected contacts

- Maintain proper hygiene of foot

CONCLUSION: Our study concludes that prevalence of onychomycosis is moderate to high in south east Rajasthan. As there are several fungi which cause the infection so it is necessary to perform culture for appropriate treatment. Males are at more risk than females to develop onychomycosis so they should maintain good personal hygiene of nails. Females are more prone to develop onychomycosis of fingernails due to frequent contact with soap and water. The newer diagnostic methods which are rapid and accurate may contribute in early diagnosis and management of infection.

\section{REFERENCES:}

1. Ahuja Sanjiv, Malhotra Shalini, Hans Charoo. Etiological Agents of Onychomycosis from a Tertiary Care Hospital in Central Delhi, India. Indian Journal of Fundamental and Applied Life Sciences. April - June 2011 ; 1 (2) :11-14

2. Prakash Gelotar, Sw ati Vachhani, Bhargav Patel, Naresh Makwana Prevalence of Fungus in Fingernail Onychomycosis. Journal of Clinical and Diagnostic Research. December $2012: 1-3$

3. Malcolm D., Richardson and David W. Wanock. Fungal Infection Diagnosis and Management. 3rd Edn. Blackwell Publishing; 2003

4. Koneman E. W. Color Atlas and Text Book of Diagnostic Microbiology. 6th edn. Lippincott Williams and Wilkins; 2006

5. Martinez PG, Nunes FG, Tomimori-Yamashita J, Urrutia M, Zarror L, Silva V and Fishman O (2009). Mycopathologia; 168: 111-116.

6. R Kaur, B kashyap, P. Bhalla. Onychomycosis- Epidemiology, Diagnosis and management. Indian Journal of Medical Microbiology. April-June 2008; 26 (2): 108-16

7. Ravinder Kaur, Bineeta Kashyap, Preena Bhalla. A five-year survey of onychomycosis in New Delhi, India: Epidemiological and laboratory aspects. Indian J Dermatol; 2007 52:39-42

8. Archana Singhal, Deepshikha Khanna. Onychomycosis: Diagnosis and management. Indian journal of Dermatology, Venereology, and Leprology. Nov.-Dec 2011;Vol 77 (6): 659-672

9. Aditya K. Gupta et.al. Non-dermatophyte onychomycosis. Dermatology Clinic; 2003:257268

10. B Sigurgeirsson, Ó Steingrímsson. Risk factors associated with onychomycosis. European Academy of Dermatology and Venereology JEADV; 2004 (18): 48-51 
11. Chander Grover, Ananta Khurana. Onychomycosis: Newer insight in pathogenesis and diagnosis. Indian journal of Dermatology, Venereology, and Leprology; May- June 2012 vol78(3) :263-270

12. Gérald Piérard. Onychomycosis and Other Superficial Fungal Infections of the Foot in the Elderly: A Pan-European Survey. Dermatology. 2001;202:220-224

Table-1

\begin{tabular}{|l|l|l|l|l|l|l|}
\hline \multirow{2}{*}{ Fungus } & \multicolumn{2}{|l|}{ Number } & \multicolumn{2}{l|}{ Male } & Female \\
\cline { 2 - 7 } & & $\mathbf{\%}(\mathbf{N 5 5}$ & & $\mathbf{\%}(\mathbf{N}=55)$ & & \% (N=55) \\
\hline Trichophyton Spp. & 28 & 50.9 & 18 & 32.72 & 10 & 18.18 \\
\hline Candida Spp. & 10 & 18.18 & 06 & 10.9 & 04 & 7.27 \\
\hline Aspergillus Spp. & 09 & 16.36 & 06 & 10.9 & 03 & 5.45 \\
\hline Scopulariopsis & 5 & 9.09 & 03 & 5.45 & 02 & 3.63 \\
\hline Fusarium & 2 & 3.63 & 01 & 1.81 & 01 & 1.81 \\
\hline Alternaria & 1 & 1.81 & 01 & 1.81 & 00 & 0 \\
\hline Total & 55 & 100 & 35 & 63.63 & 20 & 36.36 \\
\hline
\end{tabular}

Distribution of various fungi among male and female

Table- 2

\begin{tabular}{|l|l|l|l|l|}
\hline Fungus & Species & Number & Male & Female \\
\hline \multirow{3}{*}{ Tricophyton } & Tricophyton rubrum & 20 & 13 & 7 \\
\cline { 2 - 6 } & Tricophyton mantegrophytes & 7 & 4 & 3 \\
\cline { 2 - 6 } & Tricophyton tonsurans & 1 & 1 & 00 \\
\hline \multirow{3}{*}{ Candida } & Candida albicans & 7 & 4 & 3 \\
\cline { 2 - 6 } & Candida tropicalis & 3 & 2 & 1 \\
\hline \multirow{2}{*}{ Aspergillus } & Aspergillus flavus & 7 & 5 & 1 \\
\cline { 2 - 6 } & Aspergillus niger & 2 & 1 & 2 \\
\hline Scopulariopsis & Scopulariopsis Spp. & 5 & 3 & 2 \\
\hline Fusarium & Fusarium Spp. & 2 & 1 & 1 \\
\hline Alternaria & Alternaria Spp. & 1 & 1 & 00 \\
\hline Total & & 55 & 35 & 20 \\
\hline
\end{tabular}

The distribution of various species among male and females 


\section{ORIGINAL ARTICLE}

Table-3

\begin{tabular}{|l|l|l|l|l|}
\hline Age group & Male & Female & Total \\
\cline { 4 - 5 } & & & & \% (N=55) \\
\hline $0-10$ & 00 & 00 & 00 & 0 \\
\hline $10-20$ & 01 & 01 & 02 & 3.63 \\
\hline $20-30$ & 02 & 01 & 03 & 5.45 \\
\hline $30-40$ & 02 & 02 & 04 & 7.27 \\
\hline $40-50$ & 03 & 04 & 07 & 12.72 \\
\hline $50-60$ & 09 & 07 & 16 & 29.09 \\
\hline $60-70$ & 10 & 04 & 14 & 25.45 \\
\hline $70-80$ & 08 & 01 & 09 & 16.36 \\
\hline
\end{tabular}

Incidence of fungal infection among different age 